\title{
A Bionic Fish Cilia Median-Low Frequency Three-Dimensional Piezoresistive MEMS Vector Hydrophone
}

\author{
Guo-jun Hang ${ }^{1,2}$, Zhen $\mathrm{Li}^{2,3, *}$, Shu-juan $\mathrm{Wu}^{2,3}$, Chen-yang Xue ${ }^{2,3}$, Shi-e Yang ${ }^{1}$, Wen-dong Zhang ${ }^{2,3}$
}

(Received 16 October 2013; accepted 31 December 2013; published online 20 March 2014)

\begin{abstract}
A bionic fish cilia median-low frequency three-dimensional MEMS vector hydrophone is reported in this paper. The piezoresistive reasonable position was obtained through finite element analysis by ANSYS and the structure was formed by MEMS processes including lithography, ion implantation, PECVD and etching, etc. The standing wave barrel results show that the lowest sensitivity of the hydrophone is $-200 \mathrm{~dB}$ and reach up to $-160 \mathrm{~dB}$ (in which the voltage amplification factor is 300 ). It has a good frequency response characteristics in $25 \mathrm{~Hz} \sim 1500 \mathrm{~Hz}$ band. Directivity tests displayed that the hydrophone has a good "8"-shaped directivity, in which the resolution is not less than $30 \mathrm{~dB}$, and asymmetry of the maximum axial sensitivity value is less than $1.2 \mathrm{~dB}$.
\end{abstract}

Keywords: MEMS vector hydrophone; Bionic; ANSYS; Median-low frequency

Citation: Guo-jun Hang, Zhen Li, Shu-juan Wu, Chen-yang Xue, Shi-e Yang and Wen-dong Zhang, "A Bionic Fish Cilia Median-Low Frequency Three-Dimensional Piezoresistive MEMS Vector Hydrophone", Nano-Micro Lett. 6(2), 136-142 (2014). http://dx.doi.org/10.5101/nml.v6i2.p136-142

\section{Introduction}

With the development of underwater acoustical warfare, high frequency noise radiated by underwater motion platform has greatly reduced. Especially after anechoic tile using on majority of submarines, the working frequency of senor has dropped to below $3 \mathrm{kHz}$, which makes the underwater acoustic detection to the median-low frequency $[1,2]$. To obtain the spatial gain with small scale senor array at low frequency and precisely azimuth information as the underwater target, vector hydrophone is a best choice [3,4]. Mostly, traditional co-vibrating vector hydrophones adopt move coil or piezoelectric principle [5].

In recent years, the detection for underwater acoustic signals with a variety of new sensing mechanism was reported. For example, a PVDF film hydrophone made by Britain and France has been used in their submarines. Bionics is the application of biological methods and systems found in nature to the study and design of engineering systems and modern technology. The two-dimensional bionic vector hydrophone developed by North University in China is a new type of vector hydrophone, which has the advantages of small size, vector character, low-cost, easy installation, etc [6]. However, as a two-dimensional vector hydrophone, it is not useful in spatial localization. Therefore, there is an urgent need to develop a three-dimensional MEMS vector hydrophone.

In this paper, we developed a three-dimensional MEMS vector hydrophone based on fish cilia and piezoresistive principle. The detailed design, fabrication, and performance test are discussed in the following sections.

\footnotetext{
${ }^{1}$ School of Marine Science and Technology, Northwestern Polytechnical University, Xi'an 710072, China

${ }^{2}$ Key Laboratory of Instrumentation Science \& Dynamic Measurement, Ministry of Education, North University of China, Taiyuan 030051, China

${ }^{3}$ Key Laboratory of Science and Technology on Electronic Test \& Measurement, North University of China, Taiyuan 030051, China

*Corresponding author. E-mail: lizhen_nuc@163.com
} 


\section{Sensor design and analysis}

The lateral line, as a sensory organ, is peculiar to fish and amphibians. Figure 1 shows the fish lateral line which consists of cilium shape mechanoreceptors or neuromasts. It is covered with a jelly-like cupola located on the skin or in the canals along the body. When the pressure of water is changed by acoustic waves, the fluid motion gets into pore through lateral line and delivers to mucus. This will cause the flowing of mucus which makes the displacement of hair cell. Sensory cells can be stimulated and the stimulation is transmitted to medulla oblongata by nerve fiber and the fish will react according to the signal.

According to the above fish lateral line structure, a three-dimensional MEMS vector hydrophone was designed as shown in Fig. 2. The sensor hair was imitated by rigid cylinder ( $\mathrm{X}$ and $\mathrm{Y}$ direction) and long cantilever beam ( $\mathrm{Z}$ direction). The sensory cells and

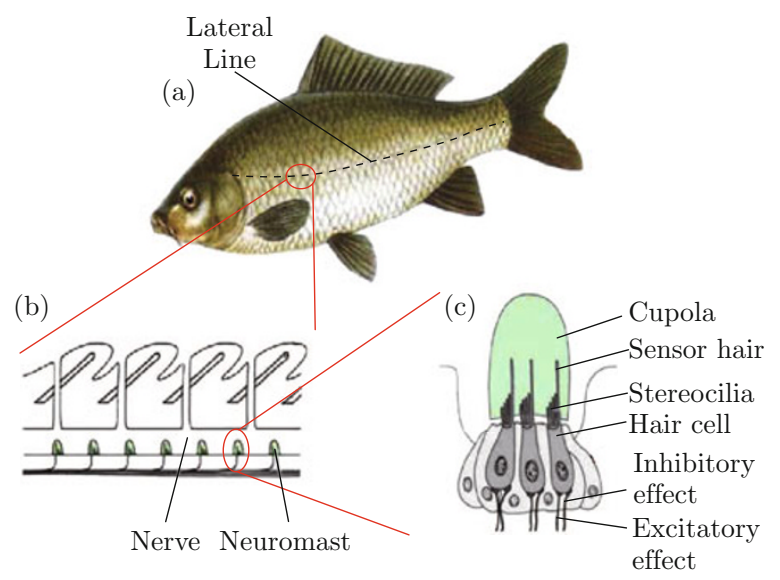

Fig. 1 Schematic drawing of fish lateral line.

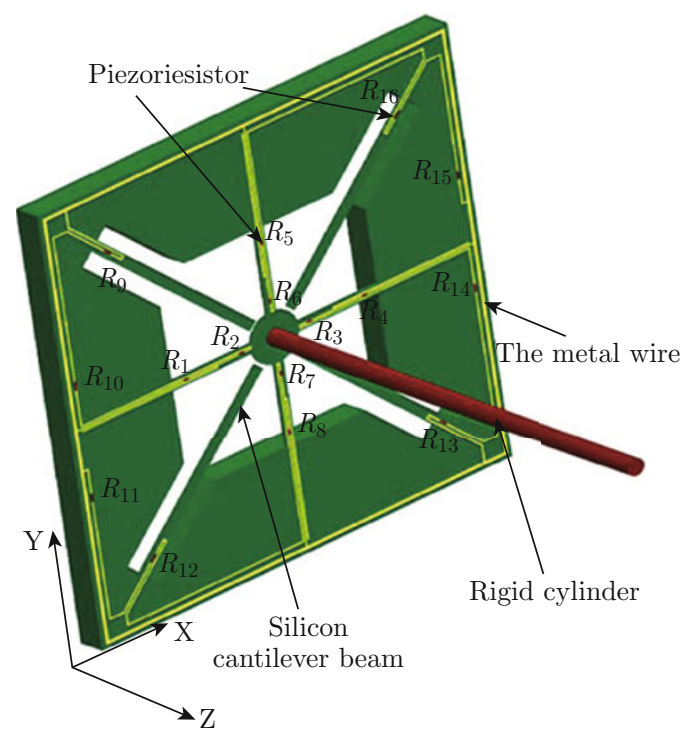

Fig. 2 Schematic of the bionic MEMS hydrophone. efferent nerve were imitated by the piezoresistors and metal lead, respectively. Moreover, the piezoresistors of $\mathrm{X}$ and $\mathrm{Y}$ direction were linked by full wheat stone bridge. Whereas, the piezoresistors on each of two cantilever beam of $\mathrm{Z}$ direction were linked by half wheatstone bridge to form two half bridge, and the signal of two half bridges was added to make up for the deficiencies of low sensitivity of the half bridge.

According to the acoustics theory, when $k \alpha \ll 1(k$ is wavenumber and $\alpha$ is the hydrophone radial size), the scattering of incident acoustic wave caused by vector hydrophone can be ignored. In the condition of far field, the vector hydrophone is equivalent to water particles, as shown in Fig. 3.

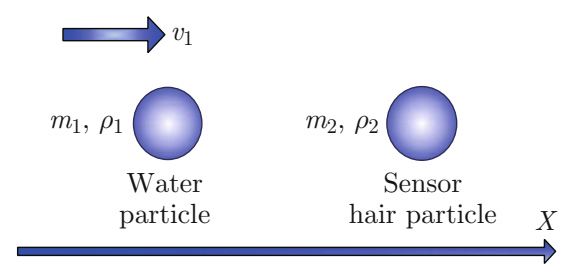

Fig. 3 Water particle with sensor hair particle.

In the acoustic perturbation, water particles begin to move and the momentum of water particles with nerve fiber is conservation in the instant of collision, that is:

$$
m_{1} \bar{v}_{1}=m_{1} \bar{v}_{1}^{\prime}+m_{2} \bar{v}_{2}
$$

where, $m_{1}$ and $m_{2}$ is the mass of water particles and nerve fiber respectively; $\bar{v}_{1}$ is the vibration velocity of water particles before the collision; $\bar{v}_{1}^{\prime}$ and $\bar{v}_{2}$ is respectively the vibration velocity of water particles and nerve fiber after the collision.

As shown in Fig. 4, the orthogonal decomposition of

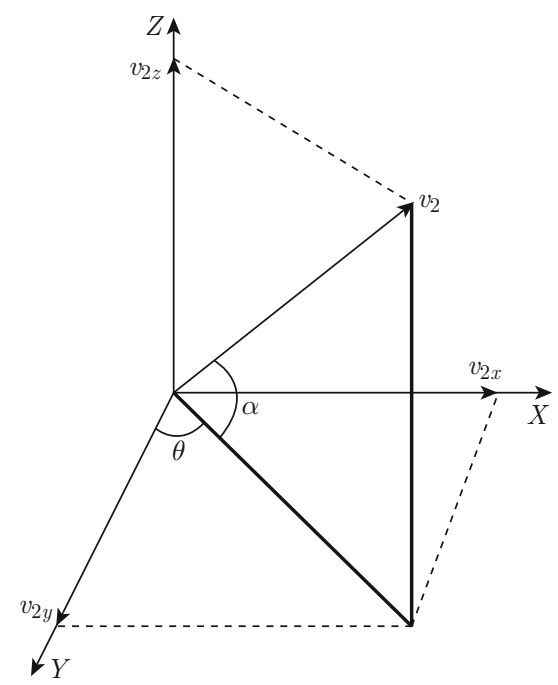

Fig. 4 Orthogonal decomposition of $\bar{v}_{2}$. 
$\bar{v}_{2}$ is $\bar{v}_{2 x}, \bar{v}_{2 y}, \bar{v}_{2 z}$,

$$
\begin{aligned}
& v_{2 x}=v_{2} \cos \theta \cos \alpha \\
& v_{2 y}=v_{2} \sin \theta \cos \alpha \\
& v_{2 z}=v_{2} \sin \alpha
\end{aligned}
$$

so,

$$
\begin{aligned}
& \theta=\operatorname{actan}\left(\frac{v_{2 y}}{v_{2 x}}\right) \\
& \alpha=\operatorname{actan}\left(\frac{v_{2 z}}{\sqrt{v_{2 x}^{2}+v_{2 y}^{2}}}\right)
\end{aligned}
$$

From all above we can come to the conclusion that the acoustic propagation characteristics can be detected by testing movement of the nerve fiber.

\section{Simulation and fabrication}

It is well known, the piezoresistive effect represents the change in electrical resistivity of a semiconductor when mechanical stress is applied. The piezoresistance expression of silicon cantilever is $\Delta R / R=\sigma_{l} \pi_{l}+\sigma_{t} \pi_{t}$. For the piezoresistors with $P$-type $\langle 110\rangle$ crystal orientation, $\pi_{l}=71.8 \times 10^{-11} \mathrm{~Pa}^{-1}$, and $\pi_{t}=-66.3 \times 10^{-11}$ $\mathrm{Pa}^{-1}$, respectively. Generally, when external force acts on the cantilever, its shear stress $\sigma_{t}$ can be neglected for far less than normal stress $\sigma_{l}$. So the mechanical sensitivity of the vector hydrophone would be expressed as: $S=71.8 \times 10^{-11} \sigma_{l} V_{i n} / P$ (where $V_{i n}$ is the input voltage, and $P$ is the sound pressure) [7]. In order to obtain a maximum sensitivity, the piezoresistor cannot be placed in the maximum stress area and the nonlinear area.

The stress of the MEMS hydrophone was analyzed using ANSYS where $1 \mathrm{~Pa}$ loads were added along the $\mathrm{Y}$ direction of the rigid cylinder and $\mathrm{Z}$ direction to the cantilever beam. The stress-contour of the structures are shown in Fig. 5(a) and Fig. 6(a), whereas, the stress curves of one beam obtained by path definition in ANSYS are shown in Fig. 5(b) and Fig. 6(b).

According to the piezoresistor distribution principle, the simulation piezoresistor distribution graph was marked in Fig. 5(b) and Fig. 6(b), where there were about $100 \mu \mathrm{m}$ from the end of cross-beam and 200 $\mu \mathrm{m}$ from the roots of silicon cantilever. Moreover, the transverse stress of cross-beam $\mathrm{X}$ direction was approximated to zero in symmetrical distribution as shown in Fig. 5 and Fig. 6.

The MEMS hydrophone microstructure was fabricated by standard MEMS process using 4-inch SOI wafers with the electrical resistivity of $2 \sim 4 \Omega \cdot \mathrm{cm}$, the active layer thickness of $20 \mu \mathrm{m}$, the buried oxide layer thickness of $2 \mu \mathrm{m}$ and handle wafer thickness of $400 \mu \mathrm{m}$. The MEMS fabrication technological processes are
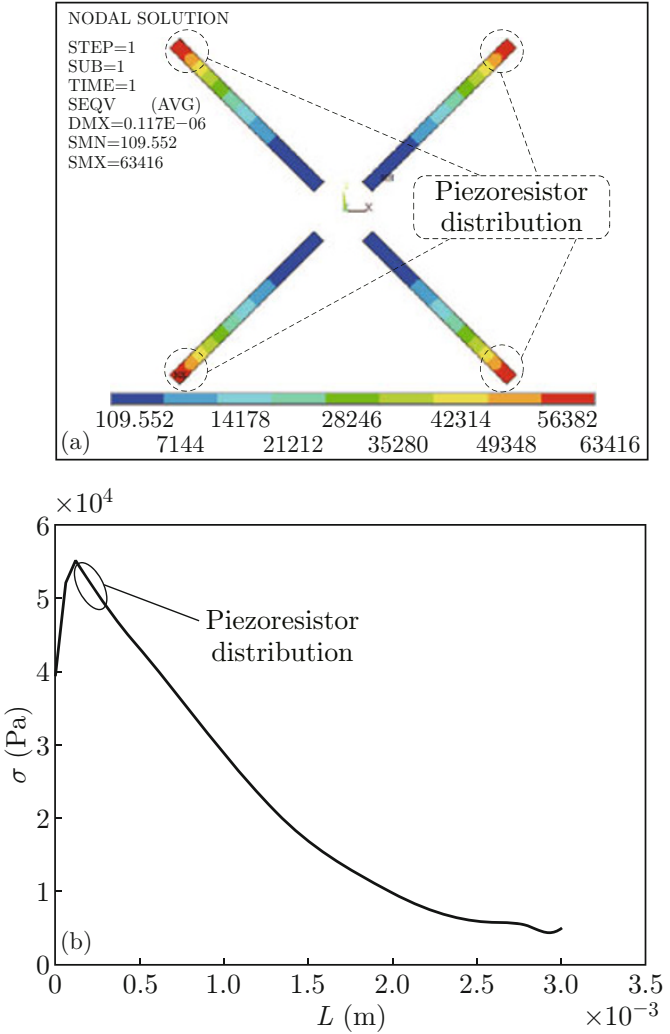

Fig. 5 (a) The stress-contour of cantilever beam; (b) Curve of cantilever stress distribution.
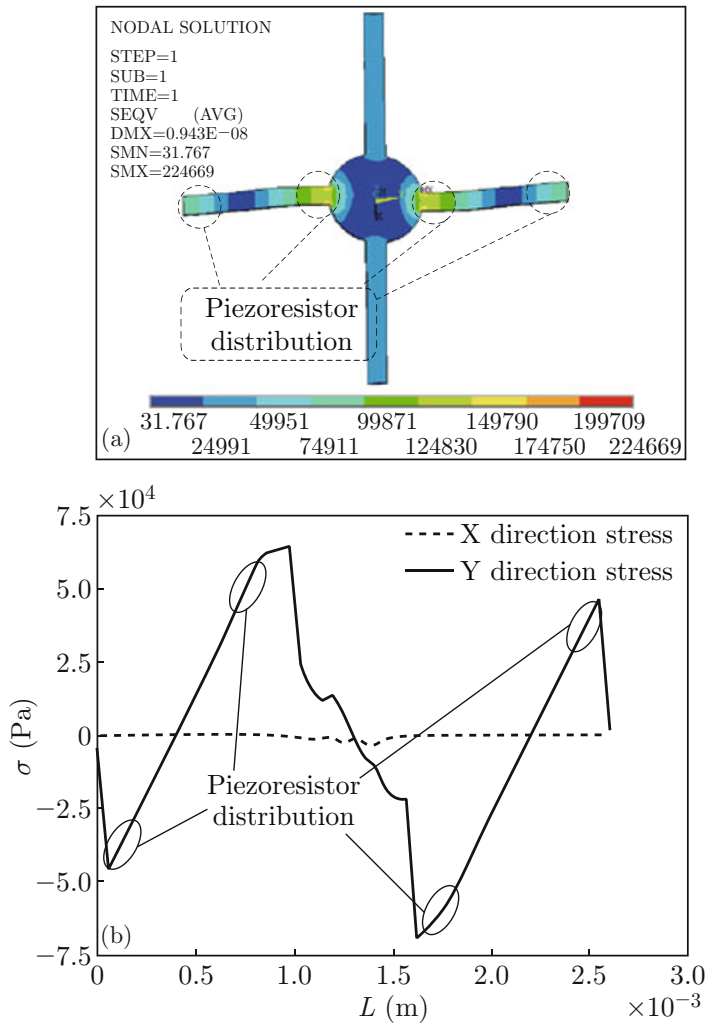

Fig. 6 (a) The stress-contour of cross-beam; (b) Curve of cross-beam stress distribution. 


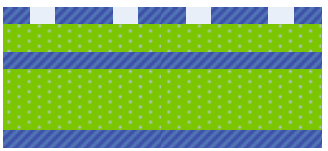

(a)

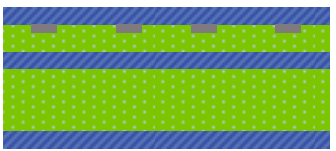

(b)

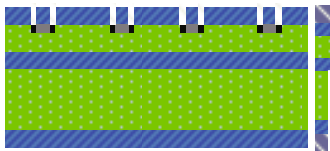

(c)

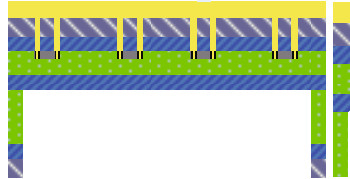

(e)

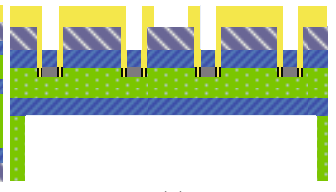

(f)

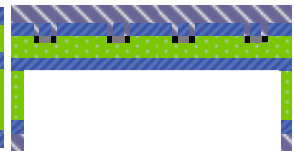

(d)

Fig. 7 The process of manufacturing technology. (a) Oxidation and ICP etching; (b) Implant boron to form piezoresistors and re-oxidation; (c) ICP etching and implantation dense boron; (d) PECVD epitaxy and ICP etching; (e) ICP etching and sputtering gold; (f) Gold etching and release the structure.

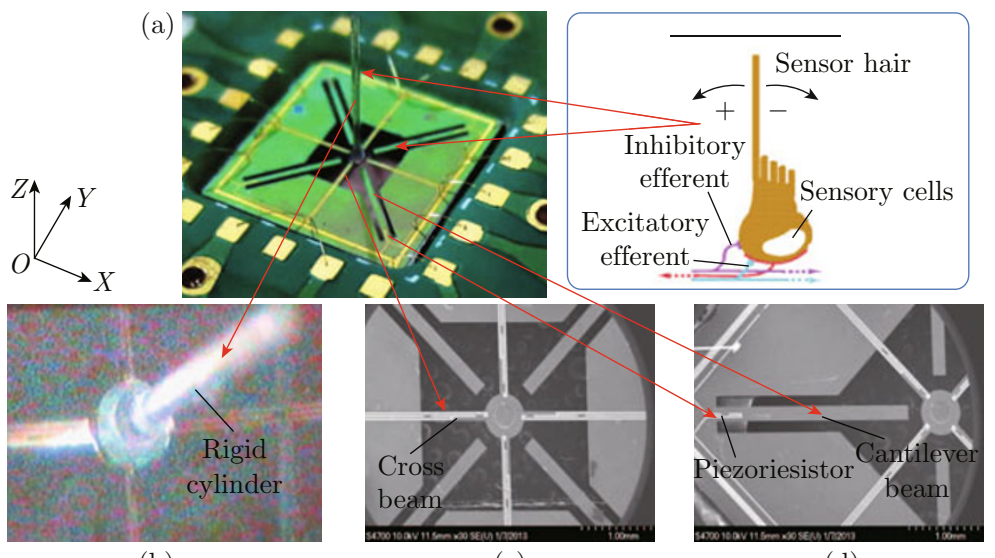

(b)

(c)

(d)

Fig. 8 Images of the hydrophone microstructure.

shown in Fig. 7, including oxidation, ICP etching, ion implantation, films growth and vapor plating processes.

\section{Bionic package and test}

Figure 8 shows the images of the hydrophone microstructure. To perform an underwater test of the MEMS vector hydrophone, the microstructure must be packaged to avoid damage. The package structure not only has a protective effect, but ensures the external sound signal to the maximum transfer to biomimetic cilia. According to the neuromast model shown in Fig. 1(c), bionic package structure was made. The bionic package structure is composed of two parts: transferal acoustic cap manufactured by the polyurethane $[8,9]$ with better acoustic properties and silicone oil which is used to fill into the acoustic cap. The detailed package structure is shown in Fig. 9.

To verify the rationality and feasibility of the hydrophone structure, the receiver sensitivity and directivity pattern of the MEMS bionic vector hydrophone was investigated in the standing wave tube calibration device. Figure 10 shows the principle of calibration device. The receiver sensitivity test adopted the antitheses calibration method compared the voltage out- put of tested MEMS hydrophone with the reference hydrophone. The test frequency is in the range of 25 $\mathrm{Hz} \sim 3.5 \mathrm{kHz}$. The vector hydrophone was fixed parallel to the acoustic wave propagation direction of the standing wave tube calibration device along direction $\mathrm{X}, \mathrm{Y}$ and $\mathrm{Z}$, respectively. Put the experiment data, which were recorded at the frequency of $1 / 3$ octave band, into the formula [10],

$$
M_{x}=M_{0} e_{x} \sin k d / e_{0} \cos k d
$$

where $M_{0}$ is the receiving sensitivity of reference hydrophone, $e_{x}$ and $e_{o}$ is respectively the open-circuit

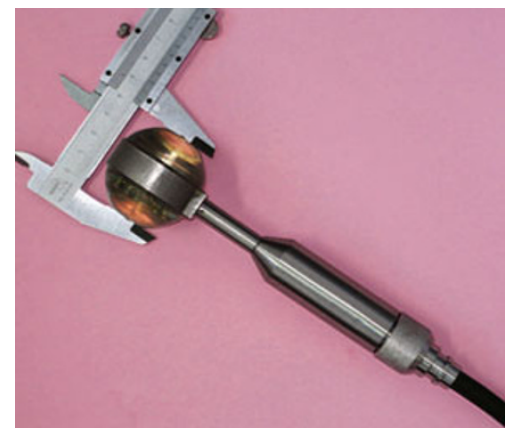

Fig. 9 Image of MEMS vector hydrophone. 


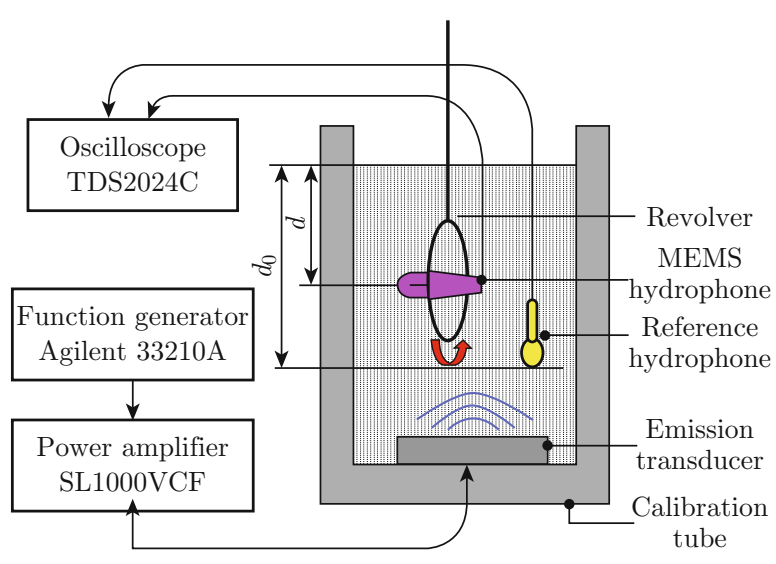

Fig. 10 The standing wave tube calibration device.

voltage of vector hydrophone and reference hydrophone, $k$ is wavenumber, and $d$ is the distance from water surface to the hydrophone. One can obtain the frequency response curve in the range of $25 \mathrm{~Hz} \sim 1.5$ $\mathrm{kHz}$ (see Fig. 11). This curve is approximated as a linear law, and sensitivity range of key direction is from $-160 \mathrm{~dB}$ to $-200 \mathrm{~dB}$ when the the voltage amplification factor is 300 .
A mechanical rotary rod was used to rotate the vector hydrophone along horizontal axis in order to change the receiving direction of sound source. We recorded the vector hydrophone sensitive in each direction. When putting the test data into the following formula [11],

$$
L=20 \log \left(e_{\theta} / e_{\max }\right)
$$

where $L$ is the normalized data, $e_{\theta}$ and $e_{\max }$ is respectively the voltage of arbitrary direction and the maximum direction of the vector hydrophone, the directivity pattern can be obtained. Figure 12 shows the directivity pattern at the frequency $500 \mathrm{~Hz}$. It can be seen there is a smooth " 8 " shape graph for the vector hydrophone. The directional resolution, which is the ratio of hydrophone axial maximum sensitivity with transverse minimum sensitivity at one certain frequency, is no less than $30 \mathrm{~dB}$.

\section{Conclusions}

A median-low frequency three-dimensional MEMS vector hydrophone is presented in this paper. The hydrophone has the advantages of small size, simple
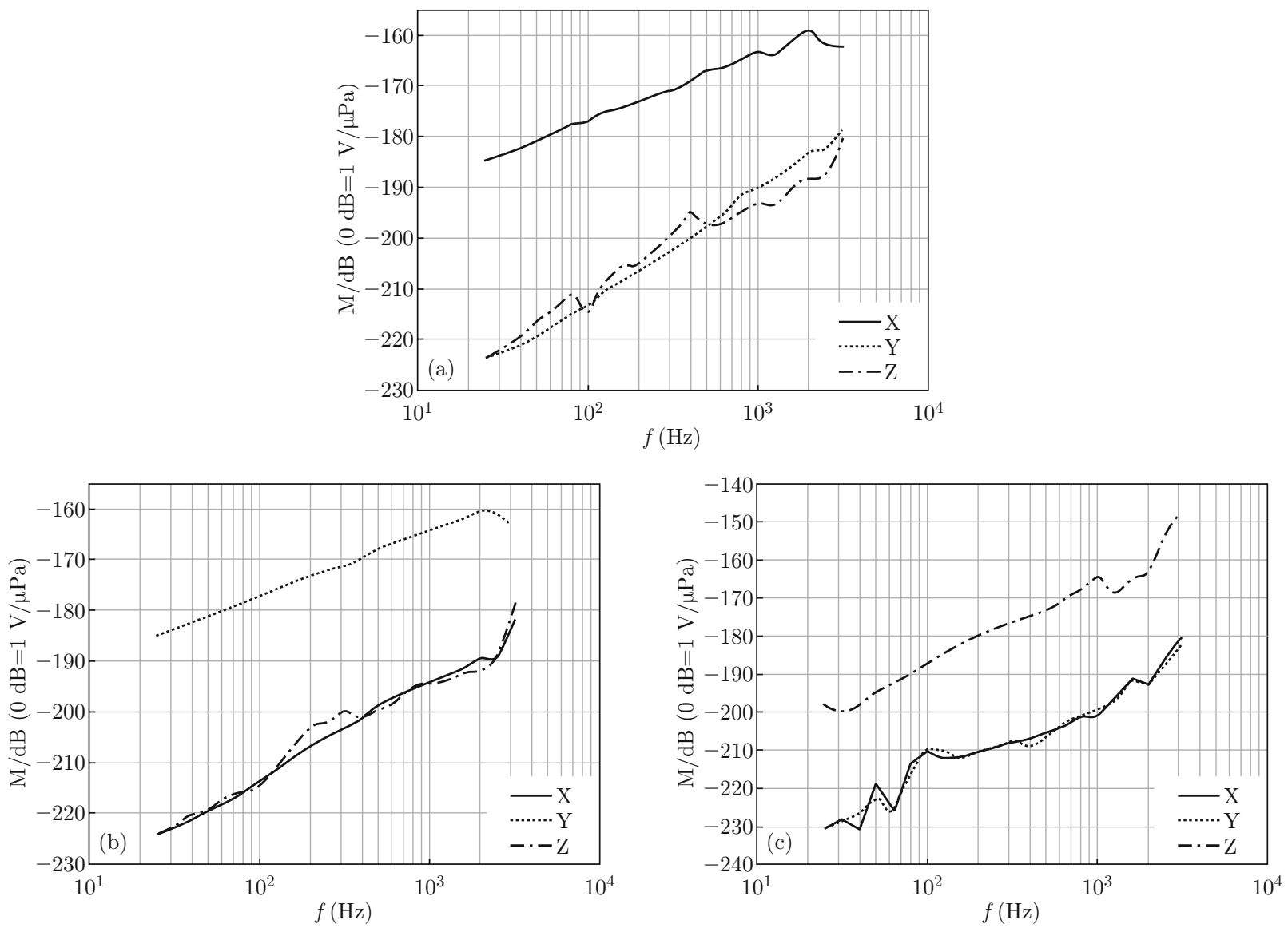

Fig. 11 (a) Frequency response curve of X direction (attenuation of $20 \mathrm{~dB}$ ); (b) Frequency response curve of Y direction (attenuation of $20 \mathrm{~dB}$ ); (c) Frequency response curve of $\mathrm{Z}$ direction (attenuation of $20 \mathrm{~dB}$ ). 


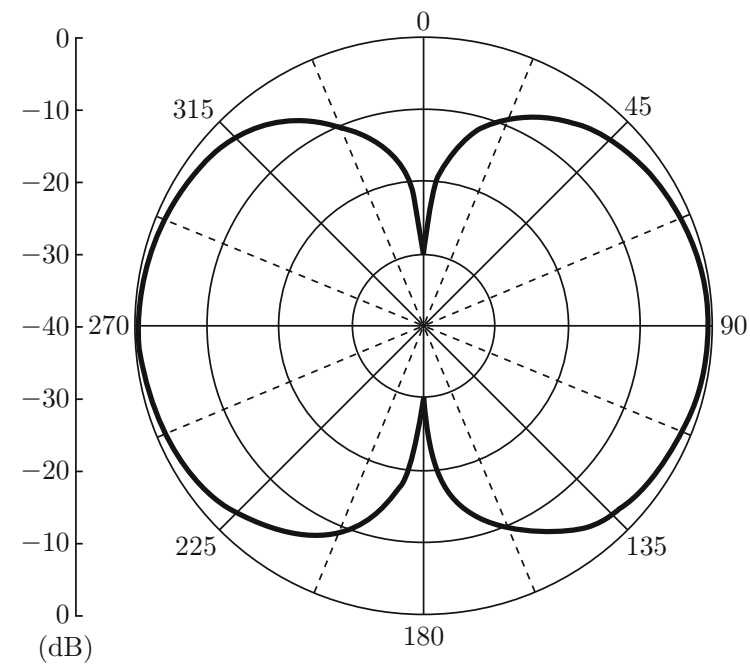

(a)

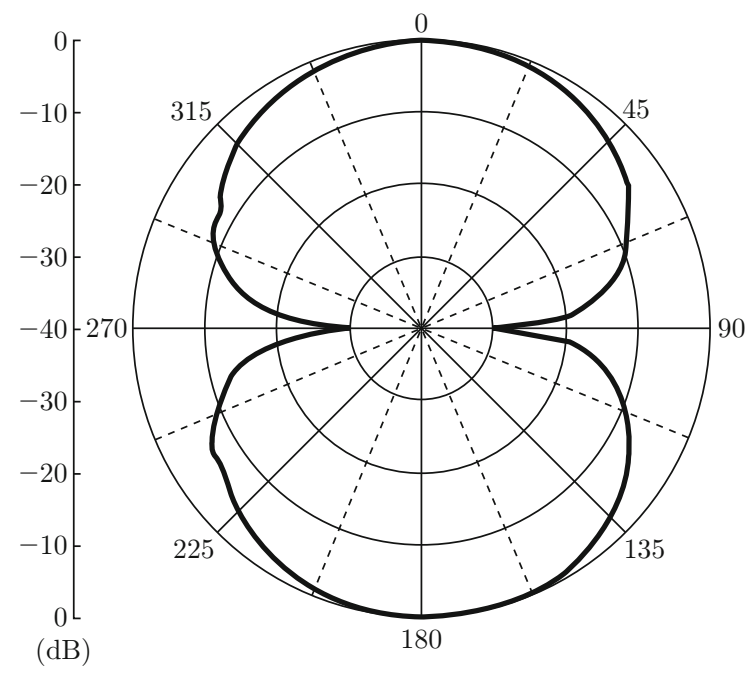

(b)

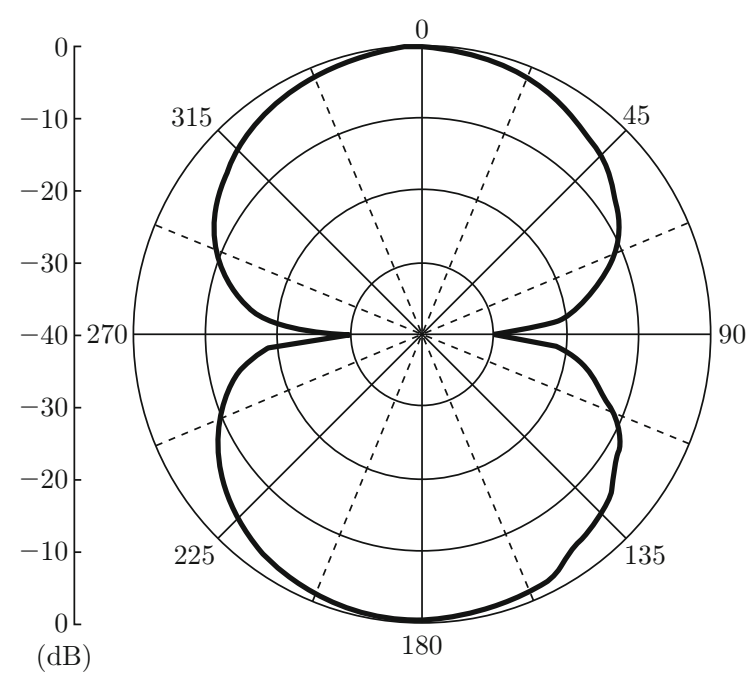

(c)

Fig. 12 (a) Directivity pattern of X direction; (b) Directivity pattern of Y direction; (c) Directivity pattern of Z direction.

manufacturing and high sensitivity. According to the test results, the hydrophone has a good frequency response in the range of $25 \mathrm{~Hz} \sim 1500 \mathrm{~Hz}$, and its sensitivity can reach up to $-180 \mathrm{~dB}$. Directivity tests displayed that the hydrophone has a good "8"-shaped directivity, whose resolution was not less than $30 \mathrm{~B}$. The improvement of the hydrophone sensitivity and expanding its available band are under ongoing.

\section{Acknowledgement}

This project is supported by the National Science Foundation of China $(51205374,61127008)$ and by Shanxi province Science foundation for Youths (2012021013-3).

\section{References}

[1] Y. Yu, Jun-ying Hui, Y. Chen, Guo-cang Sun and C.
Teng, "Research on target depth classification in low frequency acoustics field of shallow water", Acta Phys. Sin. 58(9), 6335-6343 (2009).

[2] Ian F. Akyildiz, Dario Pompili and Tommaso Melodia, "Underwater acoustic sensor networks: research challenges", Ad Ho. Networks 3(3), 257-279 (2005). http://dx.doi.org/10.1016/j.adhoc. 2005.01.004

[3] Guojun Zhang, Panpan Wang, Linggang Guan, Jijun Xiong and Wendong Zhang, "Improvement of the MEMS bionic vector hydrophone", Microelectr. J. 42(5), 815-819 (2011). http://dx.doi.org/10.1016/ j.mejo. 2011.01.002

[4] Jun-wei Zhao, Hua-wei Chen and Jin-ming Li, "Research on passive acoustics-guidance system based on vector hydrophones", J. Harbin Eng. Univ. 25(1), 2529 (2004).

[5] Li-jie Chen, Peng Zhang, Xing-ye Xu and Fu-jiang Wang, "Overview of vector hydrophone", Transducer and Micro-system Technologies 6, 5-8 (2006).

[6] Guojun Zhang, Shang Chen and Chenyang Xue, 
"Bionic encapsulation of a hair cell vector hydrophone based on MEMS", Nanotechnology and Precision Engineering 7(3), 221-225 (2009).

[7] Mohamed Gad-el-Hak, "MEMS design and fabrication", China Machine Press, Beijing, China, 49-52 (2010).

[8] Jiao Xu, Guojun Zhang, Guixiong Shi, Xiaoyao Wang, Xibao Liu and Wendong Zhang, "Advancements in encapsulation of hair vector hydrophone", Chinese Journal of Structural Chemistry 24(4), 1-5 (2011).

[9] Sungjoon Choi, Haksue Lee and Wonkyu Moon, "A micro-machined piezoelectric hydrophone with hydrostatically balanced air backing", Sens. \& Act. A 158(1), 60-71 (2010). http://dx.doi.org/10.1016/j. sna.2009.12.019

[10] V. A. Cordienko, E. L. Gordienko and A. V. Dryndin, "Absoulte pressure calibration of acoustic receivers in a vibrating column of liquid", Acoust. Phys. 40(2), 219222 (1994).

[11] Shang Chen, "Research of MEMS bionic vector hydrophone based on silicon", Dissertation for the Degree of M. Eng. of North University of China, 2008. 